Fuzzy-TOPSIS based optimal handover decisionmaking algorithm for fifth-generation of mobile communications system

Qianyu Liu, Chiew Foong Kwong, Sibo Zhang and Lincan Li

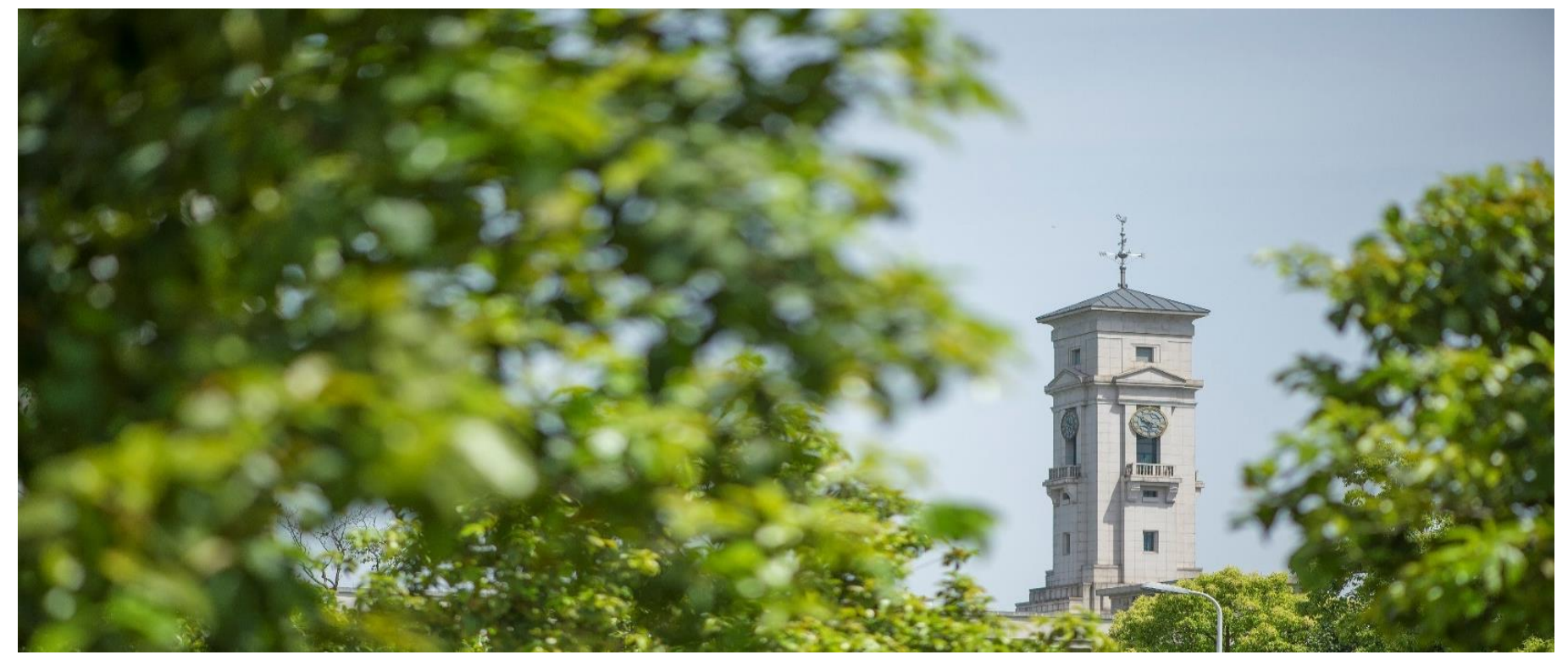


University of Nottingham Ningbo China, 199 Taikang East Road, Ningbo, 315100, Zhejiang, China.

First published 2019

This work is made available under the terms of the Creative Commons Attribution 4.0 International License:

http://creativecommons.org/licenses/by/4.0

The work is licenced to the University of Nottingham Ningbo China under the Global University Publication Licence:

https://www.nottingham.edu.cn/en/library/documents/researchsupport/global-university-publications-licence.pdf 


\title{
Fuzzy-TOPSIS based optimal handover decision-making algorithm for fifth-generation of mobile communications system
}

\author{
Qianyu Liu ${ }^{1}$, Chiew Foong Kwong ${ }^{2}$, Sibo Zhang ${ }^{2}$ and Lincan $\mathrm{Li}^{2}$ \\ ${ }^{1}$ International Doctoral Innovation Centre (IDIC), University of Nottingham Ningbo China \\ ${ }^{2}$ Department of Electrical and Electronic Engineering, University of Nottingham Ningbo China \\ Email: qianyu.liu@ nottingham.edu.cn; chiew-foong.kwong@nottingham.edu.cn;
}

\begin{abstract}
With the increasing demand for higher bandwidth and data rate of the mobile user. There are massive base stations (BS) will be deployed in the future wireless environment. Several issues could be raised dues to dense deployment of BSs, i.e. handover $(\mathrm{HO})$ ping-pong effect, unnecessary $\mathrm{HO}$ and frequent HO. To avoid these effects, the handover decision-making strategies become extremely important to select the optimal BS among all detected BS and ensure QoS for each mobile user. In this paper, the author develops a fuzzy-TOPSIS based HO algorithm to minimise the ping-pong effect and number of $\mathrm{HO}$. The proposed algorithm integrates both advantages of fuzzy logic and TOPSIS. The received signal strength intensity (RSSI) and signal to noise ratio (SNR) are considered as HO criteria in this approach. For the simulation result, the proposed $\mathrm{HO}$ algorithm can reduce ping-pong rate and a number of $\mathrm{HO}$ effectivity by comparing to conventional RSSI-based HO approach and classical multi-attribute decision making (MADM) HO method, i.e. simple additive weighting (SAW) and TOPSIS.
\end{abstract}

Index Terms - Handover management; Multi-attribute decision making; Technique for Order Preference by Similarity to an Ideal Solution; Fuzzy logic;

\section{INTRODUCTION}

To cope up with the demand of the mobile users in mobile data and the Internet of Things (IoT), the fifthgeneration of mobile communications $(5 \mathrm{G})$ system has been proposed and developed and expected to be commercialised in 2020. One of the main features in 5G is to deploy massive small base stations (BS) in the environment that provide higher capacity and coverage and thus allow ubiquitous connection for the user equipment (UE). However, due to high mobility for the future $5 \mathrm{G}$ scenario, the staying duration of UEs under each BS becomes relatively short. There will be several issues expected in the dense connection networks such as frequent handover ( $\mathrm{HO})$, unnecessary $\mathrm{HO}$ and ping-pong effect. Also, these effects can further increase communication latency and energy consumption during communication.

To mitigate these effects, $\mathrm{HO}$ needs to be triggered at the exact right moment (i.e. when?) and switch to the optimal BS (i.e. where?). Generally, the whole HO process consists of three stages: the preparation, execution and completion stage. In the preparation stage, the UE gathers $\mathrm{HO}$ related parameters such as RSSI, SNR, latency etc. of all neighbouring BSs and reports to its serving BS. The serving BS of UE will then make a decision to trigger an $\mathrm{HO}$ and select the most suitable neighbouring $\mathrm{BS}$ as $\mathrm{HO}$ target. At the execution stage, the UE will switch its connection from the serving BS to targeted BS using either hard or soft $\mathrm{HO}$ mechanism. Finally, the HO process ends with the information updates in the user plane at the $\mathrm{HO}$ completion stage. Therefore, if the selected BS is not an optimal option in terms of each HO criteria, the abnormal $\mathrm{HO}$ hence results. Based on that, it is important to adopt a suitable $\mathrm{HO}$ decision-making algorithm and $\mathrm{HO}$ criteria to ensure HO performance. The conventional HO algorithm to select BS only depends on RSSI. As such, HO is easily influenced by interference, and subsequently causing UE handover frequently among BSs that know as ping-pong effect. Furthermore, a single metric-based HO cannot meet the requirement for mobile users and the actual situation for the current or future scenario.

One of the popular approaches is to adopt multiattribute decision making (MADM) scheme to select a suitable BS. The MADM is a mathematical tool to deal with decision-making problem with multiple conflicting attributes. By applying MADM into HO decision-making stage, it can support UE to select the optimal BS as HO target among various candidate BSs concerning different attributes. Generally, conventional MADM methods are simple additive weighting (SAW), techniques for order preference by similarity for an ideal solution (TOPSIS), analytic hierarchy process (AHP) and Grey relational analysis (GRA). Among these, TOPSIS is the popular MADM variant as discussed in surveys [1]-[5].

However, the MADM in general, have some inherent drawbacks. First, the output of MADM is highly dependent on its weight value, which generally obtained from human experience. However, most of the time the mobile operators do not have full information and heavy reliance on human experience are unreliable. Apart from this, MADM itself is not able to process uncertain and imprecise data within decision criteria conclusively. In another word, when UE gather information, even with minor deviation, such as unpredictable radio signal fluctuation, the output decision from MADM are usually unreliable.

To overcome these two drawbacks in MADM, this paper proposes a hybrid of TOPSIS and fuzzy logic in HO 
decision-making, known as the fuzzy-TOPSIS. This proposal HO algorithm combines both advantages of fuzzy logic and TOPSIS, which incorporates more than one criteria as the input of $\mathrm{HO}$, and process uncertain input data and weight value to obtain the optimal decision. The fuzzy logic is implemented to process weight value and data that gathering by UE as the input for TOPSIS. Here, TOPSIS functions as the main decision-making engine in the algorithm. In addition, the proposed algorithm adopts the coefficient of standard deviation weighting techniques to calculate the degree of importance of each $\mathrm{HO}$ criteria such as RSSI and SNR. By implementing both the fuzzy logic approach and coefficient of standard deviation weighting techniques in the proposed algorithm, can effectively minimise the need of human participation, and effectively reducing human errors. The objective of the proposed HO algorithm is to decrease unnecessary $\mathrm{HO}$ and ping-pong effect during HO. The proposed algorithm will be evaluated and compared with the conventional RSSI-based algorithm and traditional MADM method i.e. SAW and TOPSIS in term of number of HOs and ping-pong effect.

The rest of this paper is organised as follow. Section 2 gives a brief literature review for MADM in HO decision making. Section 3 demonstrates the comprehensive fuzzyTOPSIS HO scheme. This scheme will be tested in a simulation environment, and its $\mathrm{HO}$ performance are shown in section 4. Finally, conclusion and future work will give in section 5 .

\section{RELATED WORKS}

Fuzzy logic is a reliable mathematical tool to trigger an $\mathrm{HO}$ as discussed in[6]-[10] The basic structure of fuzzy logic consists of fuzzification, fuzzy inference system (FIS) and defuzzification. The input parameters such as RSSI and SNR will be transformed from non-crispy format into crispy format through a group of membership functions. The crisp values will then be processed by a set of IF-THEN fuzzy rules to obtain output value. The defuzzification module will convert the crispy data into $\mathrm{HO}$ factor by another group of membership functions. The HO factor is separated from $0-1$, and 1 means $\mathrm{HO}$ with high probabilities to occur, and 0 is the least likely.

Paper [6] proposed a fuzzy logic based HO algorithm to trigger $\mathrm{HO}$ under A2 event. The fuzzy logic is implemented to adjust the HO threshold based on the quality of the channel and user's velocity. And paper [7] applied fuzzy logic to obtain optimal $\mathrm{HO}$ margin and time to trigger to minimise $\mathrm{HO}$ ping-pong effect and increase HO throughput. Paper [8] combine fuzzy logic and utility function as $\mathrm{HO}$ algorithm between WiMAX and WLAN. The fuzzy logic is used to initial HO and utility functions are then applied to select the optimal access networks. Paper [9][10] integrates artificial neuro networks into the fuzzy logic system. In this way, the fuzzy membership functions can dynamically self-adjust based on the changes of environment, which could also improve the system efficiency by reducing human intervention. Apart from $\mathrm{HO}$, the fuzzy logic are also widely used in other communications field as [11]-[13].
On the other hand, TOPSIS is also widely applied in the cell selection of $\mathrm{HO}$ as shown in papers [14]-[17]. The TOPSIS method is first developed by Hwang and Yoon [18]. The essential idea of TOPSIS is to seek for a candidate that with the shortest distance from the positive ideal solution (PIS) and with the farthest distance from negative ideal solution (NIS). Works in [14] proposed two novel TOPSIS-based HO algorithm in ultra-dense heterogeneous networks. The first algorithm adopts the entropy weighting technique to calculate weight value for each HO criteria.

In contrast, the second algorithm incorporated standard deviation weighting techniques to compute the weight value for each attribute. According to the simulation results in [14], the two proposed algorithms can reduce frequent $\mathrm{HO}$, radio link failures and enhance user throughput by comparing to existing methods. Research in [15] developed an enhanced HO decision algorithm that used the analytic networks process to weight the $\mathrm{HO}$ criteria and TOPSIS to rank the candidate networks. Reference [16] shown an improved TOPSIS HO scheme for telemedicine service to satisfy user preference in both critical and non-critical health conditions. The TOPSIS are used to deal with the patient health condition and user requirement. Authors in [17] demonstrated an optimal vertical HO approach based on TOPSIS and utility function. The TOPSIS is first applied to evaluate the performance of each access technologies based on the traffic class. Moreover, the utility function is then implemented to represent the desires of the user on the traffic class for optimal network selection. The simulation results show that the proposed approach can significantly reduce the reversal phenomenon, the ping-pong effect and number of $\mathrm{HO}$ failures.

\section{SYSTEM MODEL}

While moving, UE will collect $\mathrm{HO}$ related information for candidate BS such as RSSI, SNR, BER, etc. and report to its serving BS. The serving BS will decide the need to trigger the $\mathrm{HO}$ based on the collected information. After triggering, the UE will feed collected information to fuzzyTOPSIS HO algorithm.

The first step of fuzzy-TOPSIS HO algorithm is to build a decision matrix $\boldsymbol{D M}$ for each access networks concerning its criteria as illustrated in (1):

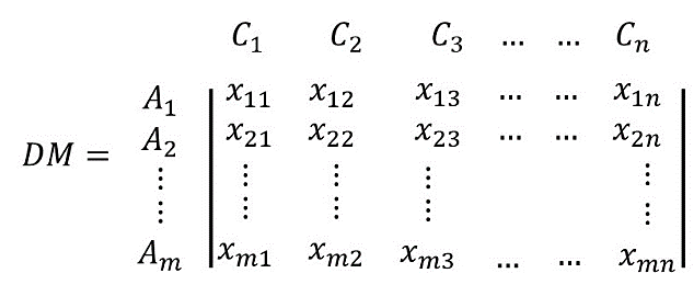

where each raw $A_{i}$ ( $i$ from 1 to $m$ ) represent one candidate $\mathrm{BS}$, and each column $\mathrm{C}_{\mathrm{j}}$ ( $\mathrm{j}$ from 1 to $\mathrm{n}$ ) perform one attribute (HO criteria). For example, $A_{1}$ is one $\mathrm{BS}$ with $n$ $\mathrm{HO}$ criteria from $\mathrm{x}_{11}$ to $\mathrm{x}_{1 \mathrm{n}}$.

Secondly, data in the matrix DM need to normalise into dimensionless by implementing Min-Max Scaling 


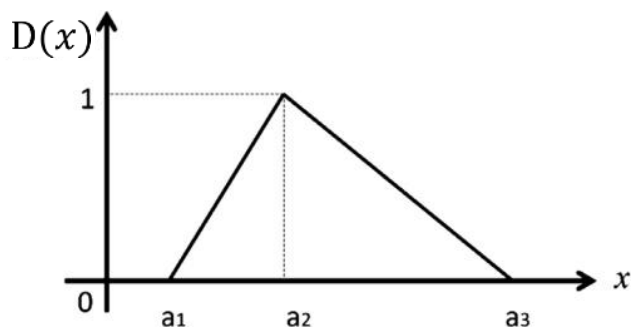

Fig. 1. Triangular Fuzzy number $D(x)$

approach for benefit and cost criteria as shown in (2)(3) respectively:

$$
\begin{aligned}
Z_{i j} & =\frac{\left[x_{i j}-\min \left\{x_{i j}\right\}\right]}{\left[\max \left\{x_{i j}\right\}-\min \left\{x_{i j}\right\}\right]} \\
Z_{i j} & =\frac{\left[\max \left\{x_{i j}\right\}-x_{i j}\right]}{\left[\max \left\{x_{i j}\right\}-\min \left\{x_{i j}\right\}\right]}
\end{aligned}
$$

After obtaining the normalised matrix, the weight value for each $\mathrm{HO}$ criteria can be calculated by the coefficient of standard deviation weighting techniques as (4) (5):

$$
\begin{gathered}
V_{j}=\frac{\sigma_{j}}{\overline{Z_{j}}} \quad(\mathrm{j}=1,2, \ldots, \mathrm{m}) \\
W_{j}=\frac{V_{j}}{\sum_{j=1}^{m} V_{j}} \quad(\mathrm{j}=1,2, \ldots, \mathrm{m})
\end{gathered}
$$

The $W j$ is the weight for criteria $\mathrm{j}$ and calculated by the coefficient of standard deviation $V j . \sigma j$ is the standard deviation of criteria $\mathrm{j}$, and $\bar{Z}_{J}$ is the average value for each criterion. The coefficient of standard deviation weighting techniques can obtain more accurate weight value than standard deviation weighting techniques.

Based on the weight for each HO criteria, the normalised decision matrix and weight value will be transformed from non-crispy values to crispy value by mapping into a triangular fuzzy membership function as shown in (6) and Fig.1. This process is known as fuzzification.

$$
D(x)=\left\{\begin{array}{rr}
0 & x \leq a_{1} \\
\frac{x-a_{1}}{a_{2}-a_{1}} & a_{1}<x \leq a_{2} \\
\frac{a_{3}-x}{a_{3}-a_{2}} & a_{2}<x \leq a_{3} \\
1 & x>a_{3}
\end{array}\right.
$$

After the fuzzification process, the normalised decision matrix DM and weight value are transformed into a normalised fuzzy decision matrix $\widetilde{\mathrm{DM}}$ and fuzzy weight array $\widetilde{W}$ as follow,

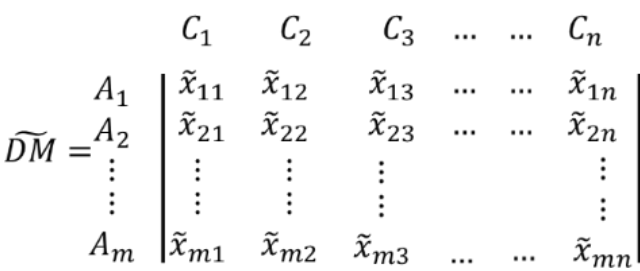

$$
\begin{aligned}
& \widetilde{W}=\left[\widetilde{w_{1}}, \widetilde{w_{2}} \ldots \ldots, \widetilde{w_{n}}\right]
\end{aligned}
$$

where, $\widetilde{x_{l j}}=\left(a_{i j}, b_{i j}, c_{i j}\right)$ represents the crispy value (fuzzy membership function) for ith candidate BS with respect to jth $\mathrm{HO}$ criteria; $\widetilde{W}_{J}=\left(a_{j 1}, b_{j 2}, c_{j 3}\right)$ indicates the crispy value of weight (the degree of importance) of each HO criteria.

Afterwards, the normalised fuzzy decision matrix $\widetilde{\mathrm{DM}}$ will multiply the fuzzy weight array $\widetilde{W}$ to obtain weighted normalised fuzzy decision matrix $\widetilde{V}$ as,

$$
\begin{aligned}
\tilde{V} & =\left|\begin{array}{cccccc}
\tilde{v}_{11} & \tilde{v}_{12} & \tilde{v}_{13} & \ldots & \ldots & \tilde{v}_{1 n} \\
\tilde{v}_{21} & \tilde{v}_{22} & \tilde{v}_{23} & \ldots & \ldots & \tilde{v}_{2 n} \\
\vdots & \vdots & \vdots & & & \vdots \\
\vdots & \vdots & \vdots & & & \vdots \\
\tilde{v}_{m 1} & \tilde{v}_{m 2} & \tilde{v}_{m 3} & \ldots & \ldots & \tilde{v}_{m n}
\end{array}\right| \\
& =\left|\begin{array}{cccccc}
\widetilde{w}_{1} \tilde{x}_{11} & \widetilde{w}_{2} \tilde{x}_{12} & \widetilde{w}_{3} \tilde{x}_{13} & \ldots & \ldots & \widetilde{w}_{n} \tilde{x}_{1 n} \\
\widetilde{w}_{1} \tilde{x}_{21} & \widetilde{w}_{2} \tilde{x}_{22} & \widetilde{w}_{3} \tilde{x}_{23} & \ldots & \ldots & \widetilde{w}_{n} \tilde{x}_{2 n} \\
\vdots & \vdots & \vdots & & & \vdots \\
\vdots & \vdots & \vdots & & & \vdots \\
\widetilde{w}_{1} \tilde{x}_{m 1} & \widetilde{w}_{2} \tilde{x}_{m 2} & \widetilde{w}_{3} \tilde{x}_{m 3} & \ldots & \ldots & \widetilde{w}_{n} \tilde{x}_{m n}
\end{array}\right|
\end{aligned}
$$

Based on this normalised fuzzy decision matrix, the fuzzy positive ideal solution $\left(A^{*}\right)$ and fuzzy negative ideal solution $\left(A^{-}\right)$are calculated by (10) (11),

$A^{+}=\widetilde{V}_{J}^{+}(j=1,2, \ldots, m) \quad$ where $\widetilde{V}_{J}^{+}=\max _{i} \widetilde{V_{l J}}$

$A^{-}=\widetilde{V}_{J}^{-}(j=1,2, \ldots, m) \quad$ where $\widetilde{V}_{J}^{-}=\min _{i} \widetilde{V_{l j}}$

The Euclidean distance from each candidate BSs to both $A^{*}$ and $A^{-}$are then calculated by (12) - (14),

$$
\begin{gathered}
d_{i}^{+}=\sum_{j=1}^{n} d\left(\widetilde{V_{l \jmath}}, \widetilde{V}_{J}^{+}\right) \\
d_{i}^{-}=\sum_{j=1}^{n} d\left(\widetilde{V_{l \jmath}}, \widetilde{V}_{J}^{-}\right) \\
d(\tilde{a}, \tilde{b})=\sqrt{\frac{1}{3}\left[\left(a_{1}-b_{1}\right)^{2}+\left(a_{2}-b_{2}\right)^{2}+\left(a_{3}-b_{3}\right)^{2}\right]}
\end{gathered}
$$

Finally, use (15) to calculates the closeness coefficient of each candidate BS to the fuzzy ideal solution,

$$
C C_{i}=\frac{d_{i}^{-}}{d_{i}^{+}+d_{i}^{-}}
$$

Thus, the candidate $\mathrm{BS}$ with the highest $C C_{i}$ are chosen as the optima BS for HO. The pseudo code for fuzzyTOSIS HO algorithm is then summarized as, 


\begin{tabular}{l|l}
\multicolumn{2}{c}{ Table 1 Simulation parameters } \\
\multicolumn{1}{c|}{ Parameters } & Specification \\
\hline BS transmitted power: & $30 \sim 35 \mathrm{dBm}$ \\
Carrier frequency: & $1.5 \sim 2 \mathrm{GHz}$ \\
Duration of simulation & $36000 \mathrm{~s}$ \\
Mobility model & Random direction \\
Number of BSs & 16 \\
The distance between each BS & $1800 \mathrm{~m}$ \\
Number of UE & Single UE \\
UE speed & $120 \mathrm{~km} / \mathrm{h}$ \\
Handover threshold & $-100.5 \mathrm{dBm}$ \\
Propagation model: & Cost-Hata model
\end{tabular}

Table 2 Fuzzy membership function transformation

\begin{tabular}{c|cc}
\hline Rank & $\begin{array}{c}\text { Criteria } \\
\text { grade }\end{array}$ & $\begin{array}{c}\text { Membership } \\
\text { functions }\end{array}$ \\
\hline Very low (VL) & 1 & $(0.00,0.10,0.25)$ \\
Low (L) & 2 & $(0.15,0.30,0.45)$ \\
Medium (M) & 3 & $(0.35,0.50,0.65)$ \\
High (H) & 4 & $(0.55,0.70,0.85)$ \\
Very high (VH) & 5 & $(0.75,0.90,1.00)$ \\
\hline
\end{tabular}

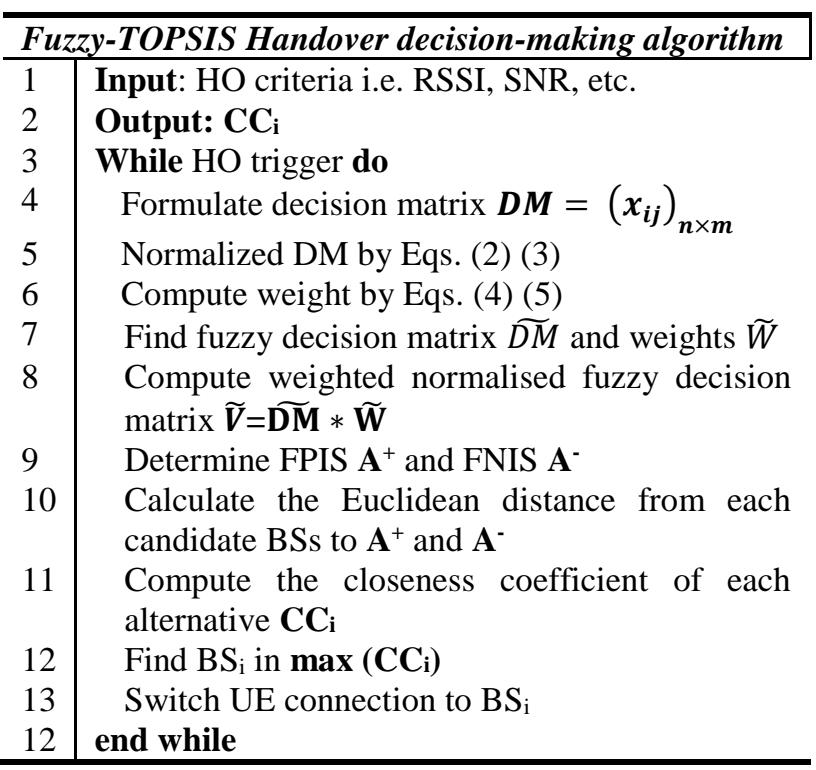

\section{PERFORMANCE EVALUATION}

\section{A. Methodology}

A simulation environment has been developed in MATLAB to test the effectiveness of the proposed algorithm. The simulation parameters are illustrated in Table 1. There are $16 \mathrm{BSs}$ are deployed in a $6000 \mathrm{~m}$ $* 6000 \mathrm{~m}$ simulation environment, and the distance between each BS is $1800 \mathrm{~m}$. A single UE is randomly moving within the simulation environment and passing through all BSs with fixed speed in $120 \mathrm{~km} / \mathrm{h}$. In addition, some HO optimisation parameter, i.e. HO margin and time to trigger are not applied in this simulation.

The RSSI and SNR are used as HO criteria in the proposed algorithm. The number of $\mathrm{HO}$ and ping-pong



Fig. 2. Triangular fuzzy membership functions

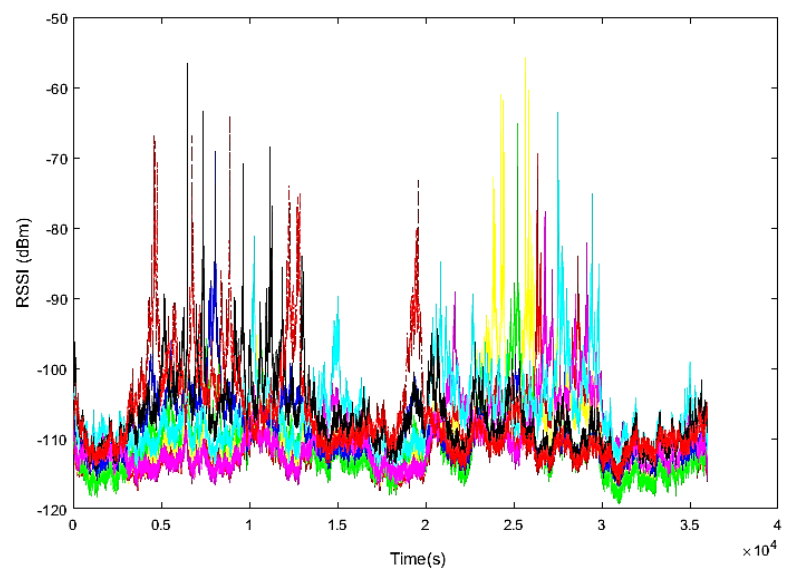

Fig. 3. RSSI of UE from each BSs

ratio are used as performance indicators to compare with SAW and TOPSIS. The ping-ping ratio is calculated as,

$$
\text { ping-pong ratio }(\%)=\frac{\text { Number of Pingpong } \mathrm{HO}}{\text { Number of } \mathrm{HO}}
$$

ping-pong $\mathrm{HO}$ in this paper is defined as when a UE is handed back to the same serving BS within 10s.

The fuzzy membership function for each $\mathrm{HO}$ criteria and weight are shown in Fig 2 and Table 2. The fuzzy linguistic variables are divided into five levels from very low to very high, and the interval for each membership function is $0.25-0.3$.



Fig.4. Performance evaluation in ping-pong ratio 




Fig. 5. Performance evaluation in ping-pong ratio

\section{B. Results and Analysis}

There are two performance indicators are adopted to evaluate the proposed algorithm i.e. HO ping-pong ratio and number of HO. The conventional RSSI-based HO algorithm, SAW and TOPSIS are chosen for comparison. Fig. 3 shows RSSI from each BSs for UE, and Fig. 4 and 5 indicate the simulation results.

According to the Fig.4, the conventional HO algorithm with the highest $\mathrm{HO}$ ping-pong ratio as it only considers RSSI as $\mathrm{HO}$ criteria. The RSSI fluctuates dues to interference that result HO becomes unstable and lead high ping-pong $\mathrm{HO}$ ratio. The conventional MADM approach SAW and TOPSIS have almost same ping-pong ratio as the same weighting approach are implemented to both methods. And the performance of the conventional MADM approach is highly related to the weight calculation approach. Owing to consideration of SNR, the performance of SAW and TOPSIS are better than conventional $\mathrm{HO}$ approach. The proposed fuzzy-TOPSIS HO algorithm with the lowest $\mathrm{HO}$ ping-pong ratio. The involvement of fuzzy logic minimises the effect of the uncertain weight value and imprecise information. With the proposed algorithm, the UE can connect to the optimal BS with less ping-pong effect. This could further result in less HO latency and ensure QoS for the user.

As shown in Fig.5, the ideal condition means no interference in the surrounding environment, which represent the theoretical minimum HO number during UE movement. The conventional approach with the highest number of $\mathrm{HO}$ as it only considered RSSI as HO criteria. The SAW and TOPSIS have the almost same HO number that much lower than conventional approach and slightly higher than the proposed algorithm. In addition, the proposed fuzzy-TOPSIS algorithm has an almost the same HO number for the ideal condition from 0 to $1500 \mathrm{~s}$. Based on that, the proposed $\mathrm{HO}$ algorithm in this paper can reduce unnecessary $\mathrm{HO}$ and frequent $\mathrm{HO}$ effectively.

\section{CONCLUSION}

In this paper, we presented on fuzzy-TOPSIS based HO decision-making algorithm for UE. Both advantages of TOPSIS and fuzzy logic are incorporated into this algorithm. To further minimise human error in human decision-making, the coefficient of standard deviation weighting techniques is adopted to calculate weight value for each HO criteria. When serving BS decide to trigger $\mathrm{HO}$, the $\mathrm{HO}$ related information such as RSSI and SNR from the neighbouring BSs will be processed by the fuzzy-TOPSIS $\mathrm{HO}$ algorithm. The algorithm will then select one optimal BS as HO target for UE.

The evaluation results show that the proposed algorithm can minimise unnecessary/frequent $\mathrm{HO}$ and ping-pong ratio effectivity that outperform conventional RSSI-based HO scheme and conventional MADM HO scheme, i.e. SAW and TOPSIS. In the future research, the proposed algorithm will involve more attributes as $\mathrm{HO}$ criteria such as bit error rates, number of resource blocks etc. In addition, more performance indicator such as HO failures, HO latency etc. will adopt to evaluate this algorithm.

\section{REFERENCES}

[1] B. R. Chandavarkar and R. M. R. Guddeti, "Simplified and improved multiple attributes alternate ranking method for vertical handover decision in heterogeneous wireless networks," Comput. Commun., vol. 83, pp. 81-97, 2016.

[2] C. H. F. Santos, M. P. S. De Lima, F. S. D. Silva, and A. Neto, "Performance Evaluation of Multiple Attribute Mobility Decision Models : A QoE-efficiency Perspective," pp. 159-166, 2017.

[3] M. Lahby, S. Baghla, and A. Sekkaki, "Survey and comparison of MADM methods for network selection access in heterogeneous networks," 2015 7th Int. Conf. New Technol. Mobil. Secur. - Proc. NTMS 2015 Conf. Work., 2015.

[4] Manisha and N. P. Singh, "Optimal network selection using MADM algorithms," in 2015 2nd International Conference on Recent Advances in Engineering \& Computational Sciences (RAECS), 2015, no. December, pp. 1-6.

[5] A. Agrawal, A. Jeyakumar, and N. Pareek, "Comparison between vertical handoff algorithms for heterogeneous wireless networks," Int. Conf. Commun. Signal Process. ICCSP 2016, pp. 1370-1373, 2016.

[6] E. Cardoso, K. Silva, and R. Francês, "Intelligent Handover Procedure for Heterogeneous LTE Networks using Fuzzy logic," pp. 2163-2168, 2017.

[7] M. Saeed, H. Kamal, and M. El-Ghoneimy, "A new fuzzy logic technique for handover parameters optimization in LTE," Proc. Int. Conf. Microelectron. ICM, pp. 53-56, 2017.

[8] A. Kammoun and N. Tabbane, "Fuzzy utility decisional vertical handover algorithm for enhancing network performances," Int. Conf. Multimed. Comput. Syst. Proceedings, pp. 337-343, 2017.

[9] C. F. Kwong, T. C. Chuah, S. W. Tan, and A. AkbariMoghanjoughi, "An adaptive fuzzy handover triggering approach for Long-Term Evolution network," Expert Syst., vol. 33, no. 1, pp. 30-45, Feb. 2016.

[10] N. Li, B. Gong, and Z. Deng, "A Handoff Algorithm Based on Parallel Fuzzy Neural Network in Mobile Satellite Networks," vol. 12, no. 7, 2017.

[11] N. Shahidah and A. H. Azni, "Multivariate Analysis for Fuzzy Correlated Node Behavior Detection in Wireless Sensor Network," vol. 13, no. 8, pp. 430-435, 2018.

[12] I. Iot and K. Sultan, "Fuzzy Rule Based System ( FRBS ) assisted Energy Efficient Controller for Smart Streetlights : An approach towards," vol. 13, no. 9, pp. 518-523, 2018.

[13] P. Sarao, "F-EEAODV : Fuzzy Based Energy Efficient Reactive Routing Protocol in Wireless Ad-hoc Networks," vol. 13, no. 7, pp. 350-356, 2018. 
[14] M. Alhabo, S. Member, L. Zhang, and S. Member, "MultiCriteria Handover Using Modified Weighted TOPSIS Methods for Heterogeneous Networks," IEEE Access, vol. PP, no. c, p. 1, 2018.

[15] M. Lahby, L. Cherkaoui, and A. Adib, "An enhancedTOPSIS based network selection technique for next generation wireless networks," Int. Conf. Telecommun., pp. $1-5,2013$.

[16] H. T. Yew, C. S. Kheau, R. K. Y. Chin, A. Chekima, and M. H. Satria, "Improved-TOPSIS based handover scheme for telemedicine service using heterogeneous wireless networks," in 2017 IEEE 2nd International Conference on Automatic Control and Intelligent Systems (I2CACIS), 2017, no. October, pp. 155-160.

[17] M. Lahby and A. Sekkaki, "Optimal vertical handover based on TOPSIS algorithm and utility function in heterogeneous wireless networks," in 2017 International Symposium on Networks, Computers and Communications (ISNCC), 2017, pp. 1-6.

[18] C.L.Hwang and K.Yoong, "Multiple Attributes Decision Making Methods and Applications," in Springer, Berlin, 1981.

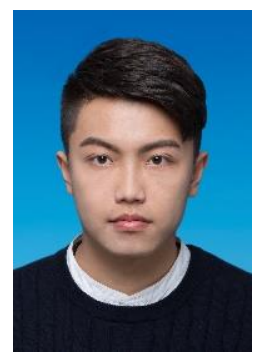

Qianyu Liu received the MSc with distinction in electronic communications and computer engineering from the University of Nottingham Ningbo China in 2017. He is currently pursuing his $\mathrm{PhD}$ degree at the University of Nottingham Ningbo China. His research interests include mobility management in $5 \mathrm{G}$ and $\mathrm{V} 2 \mathrm{X}$, handover management, and selforganisation networks.

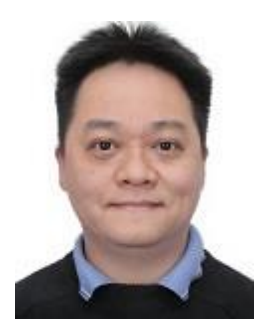

Chiew Foong Kwong received his BEng (Hons) in Electrical and Electronic Engineering from the University of Hertfordshire, UK and read MSc in Personal, Mobile and Satellite Communications, University of Bradford, UK in 2001 and 2003 respectively. He graduated his $\mathrm{PhD}$ in Wireless Communications from Multimedia University, Malaysia in 2015. He is currently an Assistant Professor at the University of Nottingham Ningbo China. His research interest includes the heterogeneous networks, mobility management for LTE and 5G, and published a number of publications in journal and conferences in this field.

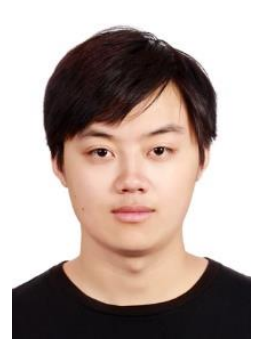

Sibo Zhang is currently an undergraduate student majoring in electrical and electronic engineering in the University of Nottingham, Ningbo China. He participated a one-year exchange programmer in the University of Nottingham, UK. He is currently working on handover management schemes.

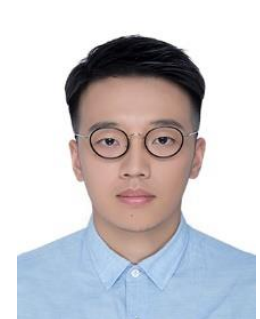

Lincan $\mathbf{L i}$ received the B.S. degree in automation from the Zhejiang University City College, Hangzhou, Zhejiang, China, in 2016, and the M.S. degree in Electronic Communications and Computer Engineering from the University of Nottingham Ningbo China, Ningbo, Zhejiang, China, in 2017.He currently is a $\mathrm{Ph} . \mathrm{D}$. in the faulty of Science Engineering in the University of Nottingham Ningbo China. His research area is about the mobility cache in the wireless communication network 